\title{
Early Improvement in Left Atrial Remodeling and Function after Mitral Valve Replacement in Organic Symptomatic Mitral Regurgitation Assessed by Three-Dimensional Echocardiography \\ Haytham Mohamed Abd El Moaty ${ }^{1 *}$, Ahmed Mahmoud Mansy ${ }^{2}$ \\ ${ }^{1}$ Cardio Thoracic Surgery Department, ${ }^{2}$ Cardiology Department, Faculty of Medicine, Al-Azhar University, Cairo, Egypt \\ *Corresponding author: Haytham Mohame Abd El Moaty, Mobile: (+2)01007217084, \\ E mail: drhaythmabdelmoaty@gmail.com
}

\begin{abstract}
Background: Mitral regurgitation increases the volume of the left ventricle and the atrium. Reverse LA remodeling is caused after surgical mitral valve replacement. There have been few studies that look at LA function following MV replacement. LA strain may be used to power LA reservoirs, conduits, and pumps. TMR causes atrial re-modeling in the left ventricle. The use of 3DE enables for more precise and dependable estimation of LA emptying fractions (total, active, and passive). In terms of estimating LA quantities, 3DE is currently more accurate than 2DE. We wanted to look at these issues.

Objectives: The current work aimed to evaluate early improvement in left atrial remodeling and function after mitral valve replacement in organic symptomatic mitral regurgitation assessed by three-dimensional echocardiography in $\mathrm{Al}$ Hussein University Hospital.

Methods: Prospective 3DE study that was performed on 60 patients with symptomatic chronic organic MR before and after surgery (valve replacement with prosthetic mechanical valve). Additionally, the maximum volume (Vol-max), lowest volume (Vol-min), and pre-contraction volume (Vol-preA) volumes of the LA were computed.

Results: Mean age of patients was $48 \pm 15$ years. Mean BMI reached $26 \pm 5 \mathrm{~kg} / \mathrm{m}^{2}$. Mean systolic blood pressure reached $123 \pm 16 \mathrm{mmHg}$ and diastolic blood pressure reached $74 \pm 8$. Male to female ratio was $1: 1$ and $51.7 \%$ of participants had a previous history of hypertension. Regarding etiology of MR, rheumatic cause was in 33.3\% of cases, mitral prolapse was the cause in most of the participants. Regarding 2DE variables of the patients, there was high significant difference between pre- and post-operative results in all variables except for LA total emptying fraction. There was no significant difference in pre and post-operative LA total emptying fraction results.

Conclusion: After a successful surgery on the mitral valve, some patients who had an organic MR and intact LV function may have a reversal of LA remodeling and functional alterations. Before surgery, a rise in active atria emptying fraction (AAEF) is linked to an increase in LV relaxation capacity. Preoperative transmitral mean gradient variation and diastolic blood pressure are factors that influence short-term postoperative reverse remodeling of the left atrium in patients.
\end{abstract}

Keywords: Left atrial function, left atrial volume, Mitral regurgitation, Mitral replacement, Three-dimensional echocardiography

\section{INTRODUCTION}

When mitral regurgitation (MR) is hemodynamically significant, the LV and LA volumes increase. Reversible remodeling of the atrium happens after successful mitral valve replacement surgery ${ }^{(1)}$. Even though surgical MV replacement has been shown to alter LA volumes in several studies, little is known about how the LA really functions. LA strains about the LA reservoir, conduit, and pump may be used to derive information about their functions. The left atrium (LA) undergoes reversible remodeling after mitral valve replacement ${ }^{(2)}$.

Due to the availability of data from threedimensional echocardiography (3DE), it is now possible to calculate LA emptying fractions with greater precision and confidence throughout the cardiac cycle (total, active, and passive). Recent research has demonstrated that $3 \mathrm{DE}$ is more accurate than $2 \mathrm{DE}$ when analysing LA volumes using magnetic resonance ${ }^{(3)}$.
Before surgery, we anticipated to see an immediate improvement in LA structure and function, even if there had been preexisting complaints.

To find out what factors are responsible for the early postoperative morphological and functional changes in the left atrium, this study sought to examine these parameters by 3DE one month following a successful surgical mitral valve replacement.

\section{MATERIAL AND METHODS}

60 patients with severe organic mitral regurgitation were studied in this prospective research. Candidates for surgical mitral valve replacement with prosthetic mechanical valve, who had symptoms, had a sinus rhythm with intact left ventricular (LV) systolic function at Cardiothoracic Surgery Department of AlHussein University Hospital during the period from June 2019 to June 2021. Pre-operative and follow up echocardiographic examinations were performed at

This article is an open access article distributed under the terms and conditions of the Creative Commons Attribution (CC BY-SA) license (http://creativecommons.org/licenses/by/4.0/) 
Cardiology Department, Al-Hussein University Hospital.

Exclusion criteria: Patients with a history of atrial fibrillation, coronary artery disease, mitral stenosis, aortic stenosis, severe aortic regurgitation, or using antiarrhythmic medications were not included. Patients having more than moderate residual postoperative MR were also excluded. Also, those who developed atrial fibrillation or ventricular arrhythmias at any stage throughout the study.

Age, gender, chronic diseases (hypertension, diabetes, heart failure, stroke, and chronic kidney disease), and smoking status were collected for all patients. A patient's anthropometric measures were taken after wearing an examination gown. A wallmounted stadiometer was used to provide an accurate measurement of height to the nearest 0.1 centimeter. On a hospital balance beam scale, the subject's weight was determined to within $0.1 \mathrm{~kg}$ accuracy. Weight $(\mathrm{kg})$ divided by height $\left(\mathrm{m}^{2}\right)$ to calculate the BMI (body mass index). Blood samples were taken from each patient, and automated blood analyzers were utilised to measure haemoglobin, total leukocyte count, and thrombocyte count.

Age, gender, body surface area, heart rate, and blood pressure were used to match patients.

All patients were subjected to a routine echocardiographic test, including Doppler 2DE and 3DE, 30 days before surgery and 30 days after surgery, as per-industry guidelines ${ }^{(4)}$. Philips ie 33 with M4S and $3 \mathrm{~V}$ transducers was used for all $2 \mathrm{DE}$ and $3 \mathrm{DE}$ testing. A second offline workstation was used to collect measurements.

Severe MR was defined as having at least two of the following characteristics: $60 \mathrm{~mL}$ or more of regurgitant volume; $0.4 \mathrm{~cm}^{2}$ or more of effective regurgitant orifice area; $0.7 \mathrm{~cm}$ or more of vena contracta width; (mean values obtained from parasternal long-axis and four-chamber views). $2 \mathrm{DE}$ and the modified biplane Simpson's rule were used to compute the LV ejection percentage ${ }^{(4)}$.

It was necessary to collect data from both tissue and standard spectral Doppler variables, such as the mitral $\mathrm{e}^{\prime}$ and $\mathrm{a}^{\prime}$ diastolic waves and the systolic pulmonary artery pressure ${ }^{(4,5)}$. Full-volume 3DE data sets were collected during an apical breath hold to assess the volume and function of the LA using electrocardiograms.

Designed software has been used to compute LA volumes and volumetric curves using automated contour tracing. The pulmonary veins and the LA appendage may need to be removed manually in certain circumstances in order to provide the best possible approximation of the LA endocardium using the automated atrial shape. After LA volumetric reconstruction, the app created volumetric curves that made it possible to quantify the volumes.
In order to compute the maximum and minimum volumes of the atrium, the following were used: maximum atrial volume (Vol-max), minimum atrial volume (Vol-min), and volume before atrial contraction (Vol-preA). Volumes indexed to body surface area were taken into account in all LA volume comparisons The following LA function indices were derived from these three volumes: Loss between LA Vol-max and LA Volmin divided by total atrial emptying fraction (TAEF); loss between LA Vol-max and LA Vol-pre A divided by TAEF is passive atrial emptying fraction (PAEF); and Loss between TAVol and LA Vol-preA divided by TAEF is active atria emptying fraction (AAEF) ${ }^{(6)}$. (Volmax before surgery - Vol-max after surgery)/Vol-max before surgery $\times 100$ was used to calculate the rate of $\mathrm{LA}$ reverse remodeling. The relative increase in AAEF was calculated using the method shown below: Postoperative AAEF/preoperative AAEF× 100. For the mean gradient variation, we used the mean gradient before surgery and subtracted it. Any rise in AAEF 30 days after surgery was recognised for the multivariate analysis.

\section{Ethical approval:}

The study was approved by the Ethics Board of Al-Azhar University and an informed written consent was obtained from each participant in the study. This work has been carried out in accordance with The Code of Ethics of the World Medical Association (Declaration of Helsinki) for studies involving humans.

\section{Statistical analysis}

IBM SPSS version 22.0 was used to analyse computer-generated data. To express quantitative data, percentages and numbers were employed. Before utilising the median in nonparametric analysis or the interquartile range in parametric analysis, it was required to perform Kolmogorov-Smirnov tests to ensure that the data were normal. We used the 0.05 significance threshold to establish the significance of the findings. The Chi-Square test was used to compare two or more groups. The Monte Carlo test may be used to adjust for any number of cells with a count less than 5. Fischer Chi-Square adjustment was applied when we compared between pre and post operative results in only two categories.

\section{RESULTS}

Regarding baseline characteristics of patients, mean age was $48 \pm 15$ years old. Mean BMI reached 26 $\pm 5 \mathrm{~kg} / \mathrm{m}^{2}$. Mean systolic blood pressure was $123 \pm 16$ $\mathrm{mmHg}$ with and diastolic blood pressure was $74 \pm 8$ mmHg. Male to female ratio was $1: 1$ and $51.7 \%$ of participants had a previous history of hypertension. Regarding Etiology of MR, rheumatic cause was in $33.3 \%$ of cases, Mitral prolapse was the cause in most of the participants (Table 1). 
Table (1): Baseline clinical characteristics of patients

\begin{tabular}{|c|c|}
\hline & Case (60) \\
\hline Age (years) & $48 \pm 15$ \\
\hline Body mass index (BMI) $\left(\mathrm{kg} / \mathrm{m}^{\wedge} 2\right)$ & $26 \pm 5$ \\
\hline Body surface area $\left(\mathrm{cm}^{\wedge} 2\right)$ & $1.8 \pm 0.2$ \\
\hline Systolic blood pressure (mmHg) & $123 \pm 16$ \\
\hline Diastolic blood pressure (mmHg) & $74 \pm 8$ \\
\hline Heart rate (beats/min) & $70 \pm 9$ \\
\hline $\begin{array}{ll}\text { Sex } & \\
& \text { Male } \\
& \text { Female }\end{array}$ & $\begin{array}{l}30(50 \%) \\
30(50 \%)\end{array}$ \\
\hline History of hypertension & $31(51.7 \%)$ \\
\hline $\begin{array}{l}\text { Etiology } \\
\text { Rheumatic } \\
\text { Mitral Prolapse } \\
\text { Other }\end{array}$ & $\begin{array}{c}20(33.3 \%) \\
36(60 \%) \\
4(6.7 \%)\end{array}$ \\
\hline
\end{tabular}

Values are expressed as mean (standard deviation) or number of patients and frequency (\%).

All 2DE patient characteristics, with the exception of the LA total emptying percentage, showed highly significant difference between their pre- and postoperative results. Neither the pre-operative nor post-operative LA total emptying fraction data showed a statistically significant change. After surgery, the LA reverse remodeling; concerning Independently Linked Variables, we found that the following factors required to be included in the multivariate model after performing independent variable analysis: diastolic blood pressure, end-diastolic diameter of the left ventricle, parasternal venal contracture, and the mean mitral gradient variation $(\mathrm{r}=-0.29 ; \mathrm{P}=0.05)$. Diastolic blood pressure before surgery and mean gradient variation $(\mathrm{r}=-0.035 ; \mathrm{P}=0.001)$ were demonstrated to be independent of Vol-max decline in the final model (LA reverse remodeling). This study found that age $(\mathrm{P}=0.23)$, high blood pressure $(\mathrm{P}=0.14)$, transapical echocardiography (TAEF) $(\mathrm{P}=0.11)$, and preoperative RVP were all linked with an increase in alphaatrioventricular (AAEF). To be certain that surgical method had no effect, we incorporated and kept transmitral mean gradient variation and surgery type in our model during the whole research period.

Table (2): Pre and Post-operative 2DE Variables of the Patients

\begin{tabular}{|c|c|c|c|}
\hline & Pre-Operative & Post Operative & P value \\
\hline LV end-diastolic diameter (mm) & $61.1(5)$ & $49(9.35)$ & $<0.0001$ \\
\hline LV end-systolic diameter (mm) & $39(5)$ & $33.07(6.26)$ & $<0.0001$ \\
\hline LV end-diastolic volume (mL) & $146.2(37)$ & $77.5(35.76)$ & $<0.0001$ \\
\hline LV end-systolic volume (mL) & $45.5(14.6)$ & $29(9.75)$ & $<0.0001$ \\
\hline LV ejection fraction & $0.72(0.05)$ & $0.6(0.06)$ & $<0.0001$ \\
\hline Mitral maximum gradient (mmHg) & $9.9(4.4)$ & $13.67(5.28)$ & $<0.0001$ \\
\hline Mitral mean gradient (mmHg) & $4.1(1.6)$ & $7(1.63)$ & $<0.0001$ \\
\hline LA maximum volume index $\left(\mathrm{mL} / \mathrm{m}^{2}\right)$ & $62.67(22.01)$ & $40.03(22.76)$ & $<0.0001$ \\
\hline LA minimum volume index $\left(\mathrm{mL} / \mathrm{m}^{2}\right)$ & $42.13(19.63)$ & $26.37(16.58)$ & $<0.0001$ \\
\hline LA pre-A volume index $\left(\mathrm{mL} / \mathrm{m}^{2}\right)$ & $51.07(21.94)$ & $35.87(22.35)$ & 0.0003 \\
\hline LA total emptying fraction & $0.35(0.11)$ & $0.44(0.37)$ & 0.073 \\
\hline LA active emptying fraction & $0.16(0.07)$ & $0.25(0.1)$ & $<0.0001$ \\
\hline LA passive emptying fraction & $0.22(0.08)$ & $0.11(0.07)$ & $<0.0001$ \\
\hline
\end{tabular}

Values are expressed as mean (standard deviation) t-test $\mathrm{P}<0.05$ is considered significant $\mathrm{p}<0.001$ is considered High significant 


\section{DISCUSSION}

A crucial aim was to reduce the vol-max following MR correction. In chronic symptomatic MR, significant LA reverse remodeling occurred as early as one month after successful MV replacement, which was due to inadequate regurgitant volume load. A decline in LA Vol-max after surgery is not exclusive to mitral valve replacement.

After mitral valve replacement, AntoniniCanterin et al. ${ }^{(7)}$ found that MR and postoperative transmitral mean gradient had a negative connection with LA volume decline six months later. This research did not find an independent relationship between postsurgery diastolic blood pressure and the decrease in LA except in univariate analysis. In univariate analysis by Marsan et $\boldsymbol{~ a l . ~}{ }^{(8)}$ age and postoperative mean gradient were only associated with LA reverse remodeling six months and one year following mitral valve surgery. Our results are in line with earlier studies. In spite of this, we discovered that one month after surgery; postoperative transmitral mean gradient enhancement was an independent variable that was negatively linked with a decrease in the left atrium volume. Because of this, valve replacement should be carried out with a hemodynamic profile that is as optimal as feasible throughout the postoperative period, as shown by the impact of LA afterload on LA remodeling. In the Cho et al. ${ }^{(9)}$ research, younger age was also linked with greater LA volume reduction following MV surgery.

Preoperative diastolic blood pressure was shown to have an independent effect on postoperative LA remodeling. These findings are in agreement with Le Bihan et al. ${ }^{(10)}$. Both the AAEF and the TAEF were improved throughout our examination. Marsan et al. ${ }^{(8)}$ found a rise in TAEF but not in AAEF when patients were referred for surgery early, when there was no LV dilatation or symptoms (1 year). Notably, in their group, the mean preoperative AAEF was $0.26 \pm 0.9$, while in our research, it was $0.11 \pm 0.07$. As a result, in their trial, AAEF could not be low enough to indicate a substantial postoperative improvement. Our findings, on the other hand, are similar with Duncan ${ }^{(11)}$ research, which found that $2 \mathrm{DE}$ enhanced AAEF in symptomatic patients with mitral valve prolapse after 6 months.

Patients with organic MR who have an intact LV ejection fraction but a poor preoperative AAEF may still benefit from significant and early postoperative improvements in active LA function, even if their AAEF is low. According to our results, diastolic and transmitral mean gradient augmentation should be carefully managed before and after surgery, as they may have a deleterious impact on LA reverse remodeling and post-surgical AAEF elevation.

\section{CONCLUSIONS}

As soon as one month following successful mitral valve surgery, even in patients who were experiencing symptoms and had an organic MR and intact LV function, early reverse LA remodeling and functional anomalies may be seen. Prior to surgery, the ability of the LV to relax had an impact on the AAEF soon after the operation. Additionally, pre-surgery baseline transmitral mean gradient variation and diastolic blood pressure are linked with short-term postoperative LA reverse remodeling.

\section{Declarations:}

Consent for Publication: I confirm that all authors accepted the manuscript for submission

Availability of data and material: Available

Competing interests: none

Funding: No fund

Conflicts of Interest: The authors declare no conflicts of interest regarding the publication of this paper.

\section{REFERENCES}

1. Avenatti E, Little S, Barker C et al. (2018): Changes in left atrial function after transcutaneous mitral valve repair. The American journal of cardiology, 122 (7): 1204-1209.

2. Deferm S, Bertrand P, Verbrugge $F$ et al. (2019): Atrial functional mitral regurgitation: JACC review topic of the week. Journal of the American College of Cardiology, 73 (19): 2465-2476.

3. Yagmur, J, Cansel, M, Kurtoglu, E et al. (2017). Assessment of left atrial volume and function by real time three-dimensional echocardiography in obese patients. Echocardiography, 34(2), 210-216.

4. Gottdiener J, Bednarz J, Devereux R et al. (2004): American Society of Echocardiography recommendations for use of echocardiography in clinical trials: A report from the american society of echocardiography's guidelines and standards committee and the task force on echocardiography in clinical trials. Journal of the American Society of Echocardiography, 17 (10): 1086-1119.

5. Mor-Avi V, Lang R, Badano L et al. (2011): Current and evolving echocardiographic techniques for the quantitative evaluation of cardiac mechanics: ASE/EAE consensus statement on methodology and indications endorsed by the Japanese Society of Echocardiography. European Journal of Echocardiography, 12 (3): 167-205.

6. Yagmur J, Cansel M, Kurtoğlu E et al. (2017): Assessment of left atrial volume and function by real time threedimensional echocardiography in obese patients. Echocardiography, 34(2):210-216.

7. Antonini-Canterin F, Beladan C, Popescu B et al. (2008): Left atrial remodelling early after mitral valve repair for degenerative mitral regurgitation. Heart, 94 (6): 759-764.

8. Marsan N, Maffessanti F, Tamborini G et al. (2011): Left atrial reverse remodeling and functional improvement after mitral valve repair in degenerative mitral regurgitation: a realtime 3-dimensional echocardiography study. American heart journal, 161 (2): 314-321.

9. Cho D, Ha J, Chang B et al. (2008): Factors determining early left atrial reverse remodeling after mitral valve surgery. The American journal of cardiology, 101 (3): 374-377.

10. Le Bihan D, Della Togna D, Barretto R et al. (2015): Early improvement in left atrial remodeling and function after mitral valve repair or replacement in organic symptomatic mitral regurgitation assessed by three-dimensional echocardiography. Echocardiography, 32 (7): 1122-1130.

11. Duncan A (2021): Transcatheter Mitral Valve Replacement: The Tendyne Device. Transcatheter Mitral Valve Therapies, 23(1): 261-275. 